\title{
A ontologia pós-estruturalista sobre liderança: identidade e materialidade em evidência
}

ELOISIO MOULIN DE SOUZA ${ }^{1}$

\author{
${ }^{1}$ Universidade Federal do Espírito SANTo (UFES) / Departamento de AdMINISTRAÇÃo, VitóRIA - ES, BRASIL
}

\begin{abstract}
Resumo
Liderança é considerada um tema relevante para os estudos organizacionais, fato que pode ser verificado pela existência de diversos periódicos acadêmicos dedicados exclusivamente ao tema. Contudo, apesar da proliferação de periódicos e várias publicações sobre o tema, a definição de liderança ainda é vaga, sendo geralmente considerada pelo mainstream um atributo masculino de líderes individuais heroicos. Assim, este artigo busca realizar uma análise crítica do mainstream sobre liderança, tendo-se como lente de análise a ontologia pós-estruturalista sobre o tema. Portanto, este artigo objetiva contribuir com o debate ontológico sobre liderança ao abordar o que é liderança para o pós-estruturalismo, enfatizando suas diferenças ontológicas em relação ao mainstream. O pós-estruturalismo promove uma ontologia de liderança alternativa ao mainstream que rompe com a concepção universal de liderança ao evidenciar sua característica microssocial e discursiva, concebendo liderança como um processo discursivo micropolítico, sendo fundamental para a entendimento da ontologia pósestruturalista de liderança compreender (1) a produção das identidades de líderes e seguidores e (2) a materialidade da liderança.
\end{abstract}

Palavras-chave: Liderança. Pós-estruturalismo. Ontologia. Identidade. Materialidade.

\section{The poststructuralist ontology on leadership: identity and materiality in evidence}

\begin{abstract}
Leadership is considered a relevant topic for organizational studies, which can be verified by the numerous academic journals dedicated exclusively to the theme. However, despite the proliferation of journals and several publications on the subject, the definition of leadership is still vague, generally considered by the mainstream to be a male attribute of heroic individual leaders. This article presents a critical analysis of the mainstream on leadership, focused on analyzing the poststructuralist ontology on leadership. The study contributes to the ontological debate on leadership by addressing what leadership is for poststructuralism, emphasizing its ontological differences in relation to the mainstream. Poststructuralism promotes an alternative ontology of leadership to the mainstream that breaks with the universal conception of leadership by highlighting its microsocial and discursive characteristic, conceiving leadership as a micro-political discursive process. It is fundamental for understanding the poststructuralist ontology of leadership to comprehend (1) the production of the identities of leaders and followers and (2) the materiality of leadership.
\end{abstract}

Keywords: Leadership. Poststructuralism. Ontology. Identity. Materiality.

\section{La ontología posestructuralista sobre liderazgo: identidad y materialidad en evidencia}

\section{Resumen}

El liderazgo se considera un tema relevante para los estudios organizacionales, un hecho que puede verificarse por la existencia de varias revistas académicas dedicadas exclusivamente al tema. Sin embargo, a pesar de la proliferación de publicaciones sobre el tema, la definición de liderazgo sigue siendo vaga, y considerada por el mainstream como un atributo masculino de líderes heroicos individuales. Así, este artículo busca llevar a cabo un análisis crítico del mainstream sobre liderazgo, teniendo como lente de análisis la ontología posestructuralista sobre el tema. Por lo tanto, este artículo tiene como objetivo contribuir al debate ontológico sobre liderazgo al abordar qué es el liderazgo para el posestructuralismo, enfatizando sus diferencias ontológicas en relación con el mainstream. El posestructuralismo promueve una ontología del liderazgo alternativa al mainstream que rompe con la concepción universal de liderazgo al evidenciar su característica microsocial y discursiva, concibiendo el liderazgo como un proceso discursivo micropolítico, haciéndose fundamental para la comprensión de la ontología posestructuralista del liderazgo entender (1) la producción de las identidades de los líderes y seguidores y (2) la materialidad del liderazgo.

Palabras clave: Liderazgo. Posestructuralismo. Ontología. Identidad. Materialidad. 


\section{INTRODUÇÃO}

O mainstream considera liderança um produto de líderes individuais que apresentam determinados traços, estilos ou comportamentos (Sutherland, Land \& Böhm, 2014). Apesar das tentativas de o mainstream delinear o que é liderança, não existe ainda uma definição clara e universal de liderança, ao ponto de Ford $(2005$, p. 237) afirmar que "there are as many (if not more) definitions of leadership as there are people who have attempted to define it". Portanto, o conceito de liderança ainda permanece ilusório e carece de definição na maioria das pesquisas desenvolvidas (Alvesson \& Spicer, 2012; Ford, 2005; Ford \& Harding, 2007; MacKillop, 2018; Sutherland et al., 2014), caracterizando-se como um conceito vago (Alvesson \& Spicer, 2012; Spicer \& Alvesson, 2011; Sutherland et al., 2014) ou um significante vazio (Kelly, 2014).

Essa realidade se aprofunda com a proliferação de novas abordagens sobre liderança nos últimos anos, trazendo novos desafios ontológicos para a definição de liderança (Dinh et al., 2014). Tais desafios demonstram que a relevância do saber acadêmico não está na reprodução de um conhecimento legitimado, mas na sua capacidade de olhar e analisar de forma crítica o conhecimento legitimado em que liderança é socialmente construída (Carroll, Firth, Ford \& Taylor, 2018). Diante do exposto, este artigo busca realizar uma análise crítica do mainstream sobre liderança, tendo-se como lente de análise a ontologia pós-estruturalista (Ford, 2005, 2010; Ford, Harding \& Learmonth, 2008; Ford, Harding, Gilmore \& Richardson, 2017; MacKillop, 2018; Sutherland et al., 2014), buscando principalmente compreender as diferenças ontológicas entre essas duas abordagens na definição de liderança e suas consequências na concepção de líder. Uma análise é considerada crítica quando "radically questions widely accepted assumptions and aims to minimize domination" (Alvesson \& Spicer, 2012, p. 376), utilizando teorias das ciências sociais (Ford \& Harding, 2007), e desafiando o conhecimento legitimado e seu modo de pensar (Alvesson \& Willmott, 2003). Assim, este artigo busca avançar a compreensão ontológica do fenômeno liderança, desafiando os conceitos mainstream naturalizados sobre o tema. As abordagens mainstream de liderança são denominadas funcionalistas/ positivistas, e privilegiam métodos quantitativos, concebendo liderança como um objeto natural que tem existência própria, à espera para ser coletado e analisado de forma objetiva (Alvesson \& Spicer, 2012; Boje, Alvarez \& Schooling, 2001; Ford, 2005). De forma geral, as abordagens funcionalistas e positivistas têm em sua ontologia a necessária conexão entre liderança e a pessoa de um líder (Sutherland et al., 2014), declarando a necessidade da existência física de um líder para que exista liderança. $O$ pós-estruturalismo nos oferece uma ontologia alternativa ao mainstream ao problematizar aspectos específicos relacionados à construção identitária de líderes e seguidores e à materialidade do fenômeno liderança.

Vale ressaltar que liderança é um tema significativo (Ford \& Harding, 2007) e frequentemente analisado pelos estudos organizacionais (Collinson \& Tourish, 2015), sendo considerada uma ferramenta capaz de resolver qualquer problema da organização (Alvesson \& Spicer, 2012) e melhorar a performance organizacional (Ford \& Harding, 2007), havendo uma carência de estudos que abordam liderança de forma crítica (Ford, 2010; Ford \& Harding, 2007). Por exemplo, Dinh et al. (2014) evidenciam que as abordagens mais utilizadas no novo milênio são a liderança carismática e a transformacional, sendo que os autores não encontraram artigos com a abordagem pós-estruturalista. Essa constatação se manifesta, ainda, nos livros mais utilizados no mundo sobre liderança (Northouse, 2016; Yukl, 2013), pois também não abordam o pós-estruturalismo em seus conteúdos (Carroll et al., 2018), evidenciando que há uma lacuna acadêmica nos estudos de liderança relacionada ao pós-estruturalismo.

Para cumprir seu intento, o próximo tópico do artigo debate as principais abordagens do mainstream sobre liderança, buscando analisar suas principais características e similaridades. Em seguida a abordagem pós-estruturalista será esmiuçada, evidenciando que liderança é um significante vazio para o pós-estruturalismo. Posteriormente, destaca-se a importância da identidade e da materialidade para se compreender a ontologia pós-estruturalista de liderança, evidenciando os princípios que regem a abordagem pós-estruturalista de liderança: crítica à visão heroica e romântica do líder; foco nas relações de poder; e repensar os seguidores. Por fim, as principais características ontológicas de liderança para o pós-estruturalismo serão abordadas. 


\section{ABORDAGENS MAINSTREAM DE LIDERANÇA}

As abordagens mainstream são denominadas positivistas (Ford, 2005) ou funcionalistas (Alvesson \& Spicer, 2012; Sutherland et al., 2014) e acreditam que os estudos de liderança devem ser conduzidos de forma similar às pesquisas realizadas pelas ciências naturais. Liderança é considerada um fenômeno que tem existência própria, um objeto que por meio de coletas sistemáticas de dados poder-se-ia chegar a uma verdade que permitiria prever o fenômeno. Essa narrativa realista considera liderança um "organizational artifact, an object-text in laboratory [...]" (Boje et al., 2001, p. 135), concebendo liderança como um "stable construct that is amenable to observation with the correct tools, which in turn will provide leaders with the techniques they need to reliably influence others" (Collinson \& Tourish, 2015, p. 578). Predomina, assim, o emprego de métodos quantitativos de pesquisa, fundamentado na crença de que a verdade sobre liderança pode ser revelada com o uso de coleta e análise de dados objetivos que permita desvendar correlações entre variáveis relacionadas à liderança (Ford, 2010). Portanto, o mainstream acredita que liderança é um fenômeno objetivo que pode ser desvendado por meio de métodos científicos quantitativos (Alvesson \& Spicer, 2012).

Para Ford (2005), existem seis abordagens mainstream sobre liderança: traços de liderança; liderança comportamental ou de estilos; liderança situacional ou contingencial; liderança transformacional e carismática; discursos teóricos de gurus; e liderança pós-heroica.

Para a abordagem de traços, liderança é um conjunto de atributos pessoais que nascem com o líder, caracterizando liderança como atributos inatos e intrínsecos do líder. Esse tipo de literatura foca em traços de pessoas históricas denominadas de Grandes Homens, estabelecendo uma visão heroica e masculinizada de liderança (Ford \& Harding, 2007; Spector, 2016), procurando identificar e descrever traços universais que definiriam liderança em qualquer situação e contexto (Ford, 2005; Yukl, 2013). Constrói-se, assim, uma visão mitológica do líder (Ford \& Harding, 2007; Kelly, 2014), a partir da qual líderes apresentam determinadas características físicas e psicológicas inatas que possibilitam o exercício da liderança (Sutherland et al., 2014), situando-a na pessoa física do líder.

A abordagem comportamental foca nas formas como líderes agem em vez de buscar estabelecer características universais inatas que definiriam quem são eles. Com isso, busca compreender os estilos de liderança e como esses estilos podem contribuir para uma liderança mais efetiva da organização (Ford, 2005), valorizando as habilidades e os comportamentos que constituem um indivíduo como líder (Sutherland et al., 2014). A liderança situacional ou contingencial salienta como o contexto dos fatores ambientais, laborais e os seguidores influenciam no exercício da liderança pelo líder (Ford, 2005). A principal premissa da abordagem situacional ou contingencial é que diferentes situações conduzem a diferentes tipos de liderança, declarando que em diferentes situações determinados estilos de liderança são preferíveis em detrimento de outros (Sutherland et al., 2014), negligenciando as relações de poder nessas análises (Collinson, 2014; Ford, 2005).

A liderança transformacional e carismática atribui importância às percepções e atribuições dos seguidores em vez de focar suas análises em traços, estilos, aspectos ambientais e laborais. O líder transformacional é responsável por criar para os seguidores uma visão de mudança organizacional que promova melhor performance organizacional (Collinson \& Tourish, 2015). Ele exerce um papel pastoral religioso, guiando os seguidores na busca por melhores resultados organizacionais por meio de seu carisma. A liderança carismática acredita que os líderes podem tornar os subordinados comprometidos com a performance organizacional, produzindo neles uma alta performance (Shamir, House \& Arthur, 1993).

Similarmente, para as teorias de gurus de liderança, o líder deve ser um guru capaz de promover uma transformação sobrenatural da organização, conduzindo-a para um nível mais elevado de sucesso. $O$ líder guru é um gestor de significados para seus seguidores, sendo considerado o redentor da organização (Ford, 2005), visando diminuir a burocracia e os níveis hierárquicos, promovendo inovação, trabalho em equipe e maior participação dos empregados (Huczynski, 1993).

As abordagens pós-heroicas ou coletivas afirmam que liderança é exercida coletivamente em vez de uma atividade exercida apenas por líderes heroicos, carismáticos, transformadores ou gurus. $O$ foco da liderança é redirecionado, passando a centrar nos seguidores, não nos líderes, dando voz a todas as pessoas da organização, considerando a inteligência coletiva de todos os membros no processo (Ford, 2005). Vale ressaltar que liderança coletiva abarca diversas abordagens de liderança cujo principal foco são nos aspectos distribuídos e compartilhados de liderança. O foco de análise deixa de ser a pessoa singular heroica de um líder e passa a ser a dimensão coletiva relacional da liderança, buscando compreender como liderança é construída a partir das interações sociais (Ospina, Foldy, Fairhurst \& Jackson, 2020). Apesar de as abordagens pós-heroicas questionarem o papel 
exclusivo do líder no processo de liderança, elas ainda mantêm uma lógica top-down. Por exemplo, o processo de liderança distribuída mantém-se fundamentado na delegação top-down de autoridade, desconsiderando, em suas análises, possíveis processos botton-up (Collinson \& Collinson, 2009). Tal processo atribui aos líderes uma posição de destaque e privilégio (Ford, 2005; Ford \& Harding, 2007), reificando-os como responsáveis em prover direção, visão e objetivos para a organização.

Contudo, apesar "these attempts to expand the notion of leadership, the assumption that it emanates from an individual (albeit 'democratic') leader remains [...]" (Sutherland et al., 2014, pp. 761-762). Assim, ao analisar as formas pelas quais os indivíduos são capazes de criar um ambiente colaborativo e delegar tarefas para outros, as abordagens coletivas ou pós-heroicas ainda colocam o líder como o catalisador primário desse processo - ou seja, líderes precedem liderança (Sutherland et al., 2014). Portanto, nessas abordagens, ainda permanece a ideia de que é necessária a existência de um líder para que exista liderança.

Apesar das diferenças entre todas as abordagens mainstream, Sutherland et al. (2014) afirmam que elas apresentam algumas características em comum: (1) liderança é produto de líderes individuais que apresentam determinadas habilidades e são responsáveis em influenciar a organização para o atingimento de alta performance; (2) concebem ontologicamente liderança como um objeto que tem existência própria e que pode ser previsto por meio de relações causais; e (3) funcionam por meio de uma crença top-down no processo de liderança em que líderes ocupam posições hierárquicas de autoridade e detêm o poder e o direito de influenciar os seguidores - estabelecem, assim, uma distinção nítida entre líderes e seguidores, estes últimos caracterizados como passivos e suscetíveis ao comportamento e estilo do líder. Em relação à concepção ontológica de liderança como um objeto, Alvesson e Spicer (2012) acrescentam que o mainstream concebe liderança como algo que tem existência independente, que pode ser localizado em uma rede de relações causais, estabelecendo liderança como um fenômeno neutro que permite promover eficácia e eficiência organizacional.

Além disso, as abordagens mainstream não consideram aspectos sociais e culturais amplos na construção do líder e não performam uma análise crítica do conteúdo produzido (Ford \& Harding, 2007). Liderança é tratada como algo neutro, uma atividade meramente técnica, não abordando poder, resistência e relações de gênero que constroem assimetrias e hierarquias sociais que afetam a construção social sobre quem é apropriado ou não para exercer liderança (Ford, 2005). Portanto, poder e resistência são silenciados pelo mainstream (Kuipers et al., 2014; MacKillop, 2018). Neste artigo, essa ontologia de liderança é problematizada pelo pós-estruturalismo ao se evidenciar a não neutralidade e as limitações dos discursos mainstream sobre liderança.

\section{LIDERANÇA NO PÓS-ESTRUTURALISMO: UM SIGNIFICANTE VAZIO}

O que diferencia o pós-estruturalismo de outras abordagens de liderança é a relevância atribuída ao discurso na compreensão de liderança. No pós-estruturalismo, "attention shifts decidedly towards an appreciation of the power of language in constituting the world, in the sense that language/discourse is taken as the means by which human actors engage, make sense of and construct the world" (Delbridge \& Ezzamel, 2005, p. 607). Discursos são "practices that systematically form the objects of which they speak [...]. [They] do not identify objects, they constitute them and in the practice of doing so conceal their own invention" (Foucault, 1974, p. 49). Portanto, linguagem não é um espelho ou uma representação da realidade; linguagem é o que constitui a realidade (Fairhurst \& Grant, 2010). Para o pós-estruturalismo, liderança é um fenômeno discursivo (Ford, 2006, 2010), enfim, "a set of changing discursive practices [...]" (MacKillop, 2018, p. 205). Ford (2006) salienta que a linguagem está localizada no discurso, e os discursos constituem uma linguagem sobre liderança, estabelecendo uma posição discursiva para um sujeito idealizado como líder (Alvesson \& Spicer, 2012), evidenciando as conexões entre discurso e poder (Foucault, 1974). Portanto, o pós-estruturalismo analisa as relações de poder situadas em contextos específicos pelas quais as práticas discursivas sobre liderança são construídas (Collinson \& Tourish, 2015; Kelly, 2014).

Para o pós-estruturalismo, liderança envolve aspectos ideológicos, políticos e discursivos (MacKillop, 2018) pelos quais o poder é disfarçado e ocultado, fazendo com que liderança atue como uma camuflagem para que o exercício do poder seja percebido como neutro, técnico e racional (Collinson \& Tourish, 2015). No mainstream, as relações de poder são camufladas por uma visão romântica e mítica do líder, privilegiando enumerar suas características universais e essenciais, prescrevendo elementos para o sucesso organizacional (MacKillop, 2018) pelos quais os interesses dos líderes e dos seguidores coadunam, onde resistência e conflito são considerados uma anomalia (Collinson \& Tourish, 2015). Opostamente, o pós-estruturalismo invoca a necessidade de desessencializar liderança (Grint, 2005), buscando salientar a complexidade das relações entre 
poder, resistência e conflito (MacKillop, 2018). Assim, poder e conflito não são considerados uma barreira ou ameaça para o exercício da liderança, mas sim como evidências da existência de diferentes alternativas e caminhos no processo de liderança (MacKillop, 2018), dinâmicas do poder que as abordagens mainstream negam (Collinson \& Tourish, 2015).

A proeminência de métodos quantitativos nas pesquisas mainstream sobre liderança constrói uma concepção de que liderança seria um conceito estático, bem delimitado e universal (Toegel \& Conger, 2002), criando "the iron cage of personality profiles" dos líderes (Ford \& Harding, 2007, p. 485), focando muito mais na compreensão do líder do que na liderança em si (Kelly, 2014; Wood, 2005). O pós-estruturalismo evita esse universalismo e essencialismo pelo qual liderança é encontrada nas qualidades pessoais ou características situacionais do líder (Fairhurst \& Grant, 2010). O perigo de se conceituar liderança como um objeto universal ou um conjunto de práticas aceitas é não reconhecer a pluralidade e a contextualidade que envolvem a liderança. Liderança é uma construção social discursiva, não um objeto natural com existência própria. É um discurso continuamente em construção sobre práticas, contextos e abordagens, não uma ciência objetiva (Carroll et al., 2018). Para o pós-estruturalismo, liderança não é um objeto tangível, nem um processo top-down entre categorias estáveis de líderes e seguidores (Kelly, 2014). Portanto, não se pode conceituar liderança como um fato, um objeto ou uma entidade, mas como "discursively, historically and contextually constituted practices (MacKillop, 2018, p. 209). Assim, liderança é resultado de uma batalha discursiva em que determinado discurso procura ser o hegemônico, fazendo com que organizações sejam compreendidas como um espaço em que discursos e estratégias competem entre si para redefinir um conjunto de consensos e alianças (MacKillop, 2018), concebendo liderança como um significante vazio (Ford et al., 2008; Kelly, 2014; MacKillop, 2018). Portanto, liderança é um conceito discursivo que adquire vida pela linguagem como um significante vazio e flutuante - ou seja, liderança "does not signify specific or fixed, but instead serves to create the conditions of possibility for many competing and complementary definitions, meanings and interpretations" (Kelly, 2004, p. 906). Significantes vazios simbolizam uma "multiplicity of contradictory demands" (MacKillop, 2018, p. 209), caracterizando liderança como uma incompletude (Kelly, 2014).

O fato de a liderança ser uma batalha discursiva com múltiplos significados a torna um significante vazio. Seja qual for a técnica ou o método utilizado para compreender liderança, sempre haverá algo que excede a sua compreensão, um "surplus of magical stuff that makes that thing what it is" (Kelly, 2014, p. 906), nunca capturando tudo o que liderança é, pois a totalidade de liderança nunca será atingida, afirmando sua incompletude, caracterizando-a como um "object whose existence is impossible but which is central to that discourse of which it is a part" (Ford et al., 2008, p. 11). Assim, o fato de a liderança ser um significante vazio explica o motivo pelo qual liderança resiste a uma definição (Kelly, 2014), tornando-se um termo vago. Liderança não tem uma única ontologia; operando como um significando vazio, adquire diversas formas, significados e ontologias (Kelly, 2014) pelos quais “the empty signifier 'leadership' provides the space for an exercise of power in the form of deciding whose interpretation matters most" (Kelly, 2014, p. 914).

Portanto, para o pós-estruturalismo, há uma necessidade de se considerar o contexto cultural e social em que a liderança se materializa, evidenciando as relações de poder pelas quais as subjetividades dos líderes e seguidores são construídas. Ford (2005, p. 242) afirma que aceitar "someone as leader or recognising leadership characteristics is as much about what we call the social and cultural context [...]", pois "a social and contextually specific (local) definition of leadership allows us to receptive to the meanings ascribed to leadership by the community employed within the organisation under study". O contexto organizacional, social e cultural molda as dinâmicas da liderança e as formas de se compreender líderes e seguidores (Collinson \& Tourish, 2015), estabelecendo liderança como um conceito que deve ser compreendido em um nível microssocial, evidenciando aspectos locais e culturais específicos do contexto em que é analisada (Ford, 2010). Por exemplo, apesar das abordagens mainstream de liderança buscarem empregar técnicas quantitativas de análise para generalizar os resultados encontrados, muitas dessas abordagens são de fato teorias domésticas, não universais, pois acabam discorrendo sobre modelos de liderança e comportamentos que valorizam e têm como referência crenças e ideologias da sociedade norte-americana, com sua ideologia focada no individualismo e no masculino como ideais de liderança (Ford, 2005). O fato de a sociedade norte-americana privilegiar o heroísmo individual faz com que as abordagens de liderança desenvolvidas nesse contexto acabem privilegiando líderes heroicos individuais (Collinson \& Tourish, 2015). Diferentes grupos tendem a definir liderança de formas conflitantes e contraditórias (Grint, 2005), demonstrando que o significado de liderança e suas práticas difere em cada espaço (MacKillop, 2018). Portanto, os significados de liderança são específicos para cada contexto cultural e social analisado. Assim, liderança e líder são realidades espacial, temporal e socialmente construídas (Alvesson, 2002) por meio de discursos que veiculam o poder, o que possibilita fazer emergir diferentes concepções e compreensões de liderança. 
Dessa forma, "poststructuralist approaches recognize the significance of context and the role and power of discourse in shaping organizational and social practices" (Ford, 2006, p. 79).

Os estudos pós-estruturalistas sobre liderança podem ser classificados em duas grandes categorias: (1) construção identitária de líderes e seguidores; e (2) materialidade corporal de liderança. A categoria construção identitária tem como foco a análise de temas sobre: (a) relações de poder e resistência nos processos de liderança; (b) fim da concepção dualista entre as identidades de líderes versus seguidores; (c) emergência de novas formas identitárias de líderes e seguidores; e (d) fragmentação dessas identidades. A categoria materialidade corporal de liderança envolve temas que: (a) analisam liderança como uma prática corporal situada e sua natureza embodied; (b) analisam identidades de gênero e seus efeitos no exercício da liderança e construção do líder; e (c) incluem práticas micropolíticas de liderança que não necessitam da figura física de um líder para ocorrer. Materialidade e identidade são aspectos fundamentais para a compreensão da ontologia pós-estruturalista de liderança, atravessados pelos princípios descritos por Collinson e Tourish (2015) que regem a abordagem pós-estruturalista: crítica à visão heroica e romântica do líder; foco nas relações de poder; e repensar os seguidores, conforme será demonstrado a seguir.

\section{A CONSTRUÇÃo IDENTITÁRIA DE LÍDERES E SEGUIDORES: FRAGMENTAÇÃo, PODER E RESISTÊNCIA}

O pós-estruturalismo desconstrói a concepção filosófica ocidental de um sujeito unificado e coerente (Ford, 2006), rompendo com a concepção unitária, essencial e universal de sujeito (Ford, 2010) e com o dualismo líder versus seguidores (Collinson, 2006). Essa concepção de sujeito pós-estruturalista irá influenciar a definição do que são líderes e seguidores, fazendo com que um dos pontos centrais para se entender liderança seja a compreensão de como o indivíduo é constituído como líder e seguidor. Vale salientar que, buscando romper com a lógica binária de pensamento, o pós-estruturalismo não separa o individual do social, como fazem as abordagens mainstream, considerando a positividade do poder na produção das identidades por meio de um processo mútuo de construção identitária (Collinson, 2006).

O pós-estruturalismo fornece novas formas de conceber identidade e poder (Collinson, 2006), ressignificando e desconstruindo categorias identitárias, evidenciando a relevância da construção de líderes e seguidores por meio da diferença (Ford, 2006). As identidades de líderes e seguidores são construídas dentro de discursos que circulam em formações discursivas e em práticas históricas locais e institucionais (Ford, 2006). Identidades são produzidas dentro de formações discursivas por estratégias enunciativas próprias situadas em lugares históricos e institucionais específicos. Enfim, identidades são formadas pelo discurso, não havendo produção de identidades fora do discurso (Ford, 2006). Dessa forma, "identities can be regarded as the meeting point in discourse and practices in which we position ourselves as the social subjects of particular discourses" (Ford, 2006, p. 79). Entretanto, o sujeito ocupa ao mesmo tempo várias posições discursivas, fazendo com que diversas identidades coexistam simultaneamente em um único sujeito (Collinson, 2006). Elementos "of these multiple identities may be overlapping and mutually reinforcing, others can be in tension and even incompatible" (Collinson, 2006, p. 183). Por exemplo, ao pesquisar líderes de uma organização do setor público, Ford (2006, p. 78) afirma que suas identidades "are complex, multifarious, contradictory and ambiguous", evidenciando a natureza fragmentada e antagônica dessas identidades. Enquanto para o mainstream as identidades dos líderes são unitárias, homogêneas coerentes e centrais (Ford, 2005), as abordagens pós-estruturalistas afirmam a "significance of multiple, contracidtory and fragmentary nature of subjectivity [...]" (Ford, 2010, p. 51). Líderes e seguidores tentam moldar ou adotar suas identidades conforme o portfólio ofertado pelos discursos hegemônicos sobre liderança que circulam (Ford \& Harding, 2007), havendo relação entre o fato de liderança ser um fenômeno discursivo com a produção discursiva dessas identidades.

Assim, são os discursos que circulam sobre liderança que ofertam as posições discursivas que podem ser ocupadas para que um indivíduo seja formado como líder ou seguidor, pois os discursos são formativos das identidades líder e seguidor (MacKillop, 2018). Portanto, o que somos depende das posições disponibilizadas pelas práticas discursivas que produzem sentidos para nossas vidas (Ford \& Harding, 2007). Contudo, as identidades produzidas são contraditórias, plurais e ambíguas, não são essenciais, unitárias e universais (Ford, 2006), não possuem uma coerência interna (Ford \& Harding, 2007), e há uma pluralidade de posições subjetivas que líderes e seguidores ocupam ao mesmo tempo, mesmo que contraditórias, mutáveis e transitórias (Ford, 2006). Para o pós-estruturalismo, nós ocupamos simultaneamente várias posições antagônicas, múltiplas, altamente ambíguas e fragmentadas de sujeitos, uma confederação de identidades contraditórias, construídas por regimes de 
poder e saber em processos de identificação e diferenciação (Collinson, 2003, 2006) pelos quais "contradictory and multiple discourses construct the leadership identities [...], and these identities are simultaneously compelling and coercive, fluid and constraining" (Ford, 2006, p. 82).

Portanto, identidade nunca é um produto finalizado ou acabado, pois as posições subjetivas ofertadas pelo discurso podem ter duração longa ou temporária, fazendo com que "identities are in constant flux, depending on the changing positions we take up or resist" (Ford, 2006, p. 79), o que as torna sempre instáveis e divididas, principalmente pelo fato de que o discurso conecta as palavras líder, seguidor e liderança com a história, a sociedade e a cultura (Ford \& Harding, 2007). Para o pós-estruturalismo, não existe um self a priori ou pré-social, pois nos tornamos sujeitos somente se ocuparmos as posições subjetivas disponibilizadas pela linguagem com seus significados sociais (Ford, 2006), desafiando a concepção essencial, unificada e coerente de líder e seguidor presente no mainstream.

Além disso, o pós-estruturalismo salienta várias formas identitárias de seguidores que não se caracterizam como dóceis ou domesticadas, havendo sempre a possibilidade de emergir identidades que agem de forma ativa, mesmo em posições subordinadas, fazendo com que as fronteiras líder-seguidor tornem-se permeáveis e instáveis. Essa interdependência líder-seguidor faz com que o exercício do poder não aconteça somente no sentido top-down, tão presente no mainstream (Collinson \& Tourish, 2015; Sutherland et al., 2014). Existe sempre a possibilidade de mudanças de direção do fluxo de poder, contradições e contestações (Collinson, 2011), rompendo com o dualismo líderes versus seguidores que tende a negar aos seguidores poder, agência e autonomia. O mainstream acredita que seguidores devem ser dóceis e que qualquer forma de resistência é algo ruim para a liderança, considerando dissenso e resistência de seguidores algo disfuncional. Para o pós-estruturalismo resistência, conflito e dissenso dos seguidores são importantes, pois têm o potencial de fornecer um feedback útil da liderança exercida (Collinson \& Tourish, 2015). Portanto, a dinâmica da construção das identidades dos seguidores é mais complexa do que o mainstream descreve, devendo-se inclusive considerar que muitos líderes também ocupam a posição de seguidores e encontram-se em situação de subordinação a outros líderes dentro da organização (Ford, 2005). Líderes não são agentes livres das relações de poder "who enjoy the power and of their positions, but people with complex identities that contain within them forms of coercion and control over the leadership self" (Ford, 2010, p. 52), declarando que poder e agência não são propriedades dos líderes (Collinson \& Tourish, 2015). No pós-estruturalismo, a relação líder-seguidor é interdependente, não assimétrica, pois liderança "is co-constructed, a product of sociohistorical and collective meaning making, and negotiated on an ongoing basis through a complex interplay among leadership actors, be they designed or emergent leaders, managers and/or followers" (Fairhurst \& Grant, 2010, p. 172).

Dessa forma, a ação de líderes e seguidores não é voluntarista, nem determinista, pois deve reconhecer "the importance of both structures (avoiding voluntarism) and agency (avoiding determinism)" (Collinson, 2006, p. 180). Resistência é exercida por vozes múltiplas de identidades fragmentadas e não por categorias essenciais e unificadas de líderes e seguidores (Thomas \& Davies, 2005a). Resistência opera no pós-estruturalismo no nível microssocial de subjetividades e significados (Thomas \& Davies, 2005b) em vez de em uma reação macrossocial a um poder repressivo. Assim, resistência opera de forma sutil, oculta e multidirecional no nível microssocial, não operando de forma linear e unidirecional (Thomas \& Davies, 2005b). Thomas e Davies (2005a) afirmam que não existe resistência derivada de uma perspectiva essencialista de opressão de identidades, estabelecendo resistência como práticas micropolíticas. Resistência "arising from the micro-level negotiations taking place between the subject positions offered within this script (and others), which may result in adaptation or re-writing of the script" (Thomas \& Davies, 2005a, p. 717). Assim sendo, resistência opera em menor escala em nível local, objetivando desestabilizar verdades, desafiando discursos e subjetividades normalizadas. O pós-estruturalismo privilegia análises locais das relações entre subjetividades e resistências em vez de grandes projetos emancipatórios universais e revolucionários. Seu foco é nas formas discursivas de resistência que desafiam discursos normalizantes e injustos, buscando ressignificar e desconstruir subjetividades e significados considerados naturais (Thomas \& Davies, 2005a).

Resistência não significa uma ação ou um comportamento contra um poder opressor, mas a produção de novos significados e subjetividades. Para o pós-estruturalismo, resistência deve ser compreendida e analisada na produção de significados que ofertam diferentes formas identitárias e subjetivas pelas quais "individuals struggle to create, appropriate and transform discourse [...]" (Thomas \& Davies, 2005b, p. 684). Seguidores e líderes não são meros recipientes passivos dos discursos que os formam como sujeitos, e todos são capazes de exercer micropolíticas de resistência (Thomas \& Davies, 2005b). Mesmo sendo construídos pelo discurso, os indivíduos têm um self resistente que deriva do embate entre as posições subjetivas contraditórias e as práticas sociais (Thomas \& Davies, 2005a). Portanto, resistência "is understood as a constant process of 
adaptation, subersion and reinscription of dominant discourses" fomentada "by the contradictons, weakness and gaps, between alternative subject positions" (Thomas \& Davies, 2005b, p. 687). Dessa forma, a análise da resistência no pós-estruturalismo tem como foco a ressignificação das identidades e dos significados (Thomas \& Davies, 2005b).

Outro aspecto relevante para a compreensão da ontologia pós-estruturalista é a materialidade da liderança. A centralidade do corpo físico do líder para que exista liderança e a corporeidade generificada do líder são problematizados pelo pós-estruturalismo. Assim, o próximo tópico irá abordar as relações entre gênero e liderança para posteriormente abordar por que para o pós-estruturalismo liderança não depende da existência física do líder para existir.

\section{A MATERIALIDADE CORPORAL DE LIDERANÇA}

O sujeito concebido pela tradição filosófica ocidental é um sujeito sem corpo, pois na busca por um sujeito universal desconsidera aspectos corporais na constituição do líder, como gênero e raça. Influenciadas por esta tradição as teorias mainstream não consideram a importância de aspectos corporais na liderança, considerando líderes como "sites of disembodied traits, characteristics and abilities" (Ford et al., 2017, p. 1553), considerando liderança uma commodity que pode ser agregada a um indivíduo (Carroll et al., 2018). Liderança, assim, é concebida como um produto de indivíduos que apresentam determinados traços, comportamentos ou estilos (Sutherland et al., 2014). Entretanto, essa visão universal de liderança faz com que líderes sempre sejam "described and represented by somebody or something else" (Kelly, 2014, p. 906), desconsiderando aspectos identitários generificados que afetam a liderança, construindo o líder com apenas um único rosto, que é a do homem branco heroico heterossexual norte-americano.

Contrariamente, o pós-estruturalismo afirma que liderança é uma prática corporal materialmente situada, afetada por lugares, discursos e relações de poder em que se materializa: liderança "is embodied - leadership is practiced through and between bodies [...]" (Pullen \& Vacchani, 2013, p. 315), existindo inclusive corpos abjetos para o exercício da liderança. Assim, liderança há uma natureza embodied que afeta sua materialidade (Pullen \& Vacchani, 2013). Liderança é algo materialmente generificado que "embraces materiality, embodiment and corporeality" (Ford et al., 2017, p. 1553), e o gênero é utilizado para criar sentidos e significados sobre quem pode ser reconhecido como líder. Vale ressaltar que as abordagens mainstream também tratam de relações entre gênero e liderança. Entretanto, a maioria desses estudos versa sobre aspectos comportamentais de gênero e suas relações com os estilos de liderança de mulheres e homens, buscando principalmente compreender as possíveis diferenças e similaridades de estilos de liderança entre homens e mulheres, e como tais estilos influenciam na performance organizacional (Aarum \& Hansson, 2011; Ayman, Korabik \& Morris, 2009; Eagly \& Johannesen-Schmidt, 2001; Gipson, Pfaff, Mendelsohn, Catenacci \& Burke, 2017). Ford (2006, 2010) destaca que, em relação a esses estudos mainstream, o pós-estruturalismo permite uma interpretação mais subjetivista de liderança, analisando nos discursos proferidos as complexas relações entre gênero, psique e self. Evidencia, assim, que liderança funciona de forma diferente entre homens e mulheres, desvendando as relações de poder que estabelecem o domínio masculino pelo qual mulheres são consideradas líderes inapropriados ou sem credenciais (Marshall, 2007).

No mainstream prevalece um modelo patriarcal de liderança que mantém em funcionamento todo um dispositivo andrógeno que privilegia a construção da imagem do líder e de liderança como sendo de homens super-heroicos e de comportamentos masculinos (Collinson \& Tourish, 2015; Ford, 2010), perpetuando concepções hegemônicas unitárias de subjetividade e identidade que mantêm o androcentrismo nas organizações (Ford, 2006). Esses discursos reproduzem assimetrias de gênero nas organizações, valorizando a masculinidade. Isso faz com que a literatura mainstream seja mais uma literatura sobre masculinidades, reforçando o individualismo, a competitividade, a agressividade, a lógica racional, a manipulação e o controle como práticas essenciais, verdadeiras e legítimas para o exercício da liderança (Ford, 2005, 2010). Destaca-se que, para o pós-estruturalismo, não somente líderes têm um corpo generificado, mas também seus seguidores (Collinson \& Tourish, 2015), demonstrando que a assimetria de gênero se expressa quando o mainstream atribui ao líder um papel dominante, portanto masculino, enquanto seguidores são descritos como subordinados e passivos - ou seja, uma expressão do feminino (Collinson, 2006).

No mainstream, liderança é uma atividade exercida por homens brancos heterossexuais (Collinson \& Tourish, 2015; Marshall, 2007), reproduzindo desigualdades de gênero históricas (Marshall, 2007). Esse androcentrismo presente no mainstream faz 
com que as experiências masculinas sejam consideradas as de todos os líderes, concebendo a teoria sobre liderança como uma teoria masculina em que líderes são considerados indivíduos "with male stereotypic powers, atitudes and obligations" (Ford, 2005, p. 243), reificando o masculino como líderes, gestores e trabalhadores ideais (Ford, 2005), e estabelecendo o corpo masculino como modelo ideal natural de liderança, ao mesmo tempo que produz a inteligibilidade de que o masculino é um corpo neutro. Nesse processo, o líder é disembodied e constituído como um ser sem corpo, descrevendo o líder ideal como "a disembodied and rational figure, one which fits more closely with the cultural images of masculinity rather than femininity" (Ford, 2006, p. 81).

A concepção de liderança como um atributo naturalmente masculino, por um lado, estabelece os líderes como heróis (Collinson \& Tourish, 2015), mas nunca heroínas (Fletcher, 2004), naturalizando as premissas de que liderança é algo masculino (Davies, Yarrow \& Syed, 2020; Ford, 2010). Por outro lado, enquanto homens são concebidos disembodied, mulheres são constituídas como possuidoras de um corpo e o Outro dessa relação. Características femininas são consideradas uma ameaça para a organização e necessitam ser combatidas para que a organização permaneça racional, objetiva e competitiva. Consequentemente, a presença de mulheres líderes faz com que estratégias individuais e coletivas sejam utilizadas tanto por homens como por mulheres para restabelecer a ordem "neutra" de gênero ameaçada (Gerhardi, 1995). Mulheres procuram performar o masculino para se passar por um dos "meninos", um "homem honorário", adotando estilos masculinos de liderança (Ford, 2006). Isso faz com que diversos trabalhos pós-estruturalistas (Calás \& Smirchich, 1991; Collinson, 2006; Ford, 2005, 2010; Ford et al., 2017; Marshall, 2007) busquem romper com essa reprodução das assimetrias de gênero, evidenciando aspectos corporais e identitários de gênero em seus trabalhos, compreendendo liderança como um processo social mais amplo (Ford, 2010).

Materialidade não se refere somente a gênero, mas também às formas pelas quais a liderança é materializada e constituída em determinado contexto. O mainstream acredita que liderança só se materializa com a existência de um líder. Assim, somente há liderança se houver líderes, e uma organização sem líderes é uma organização sem liderança (Sutherland et al., 2014). Estudar liderança é estudar indivíduos que apresentam características consideradas essenciais para moldar a relação líder-seguidores, seja por meio de seus traços, comportamentos ou carisma individual, fazendo com que as abordagens mainstream foquem muito mais na compreensão do líder do que na da liderança em si (Kelly, 2014). O pós-estruturalismo evita o privilégio do líder na concepção de liderança (Thomas \& Davies, 2005a), considerando liderança um fenômeno social, não individual (Kelly, 2014), pelo qual a materialidade de liderança é definida como um processo em vez de um fenômeno dependente exclusivamente da existência individual de um líder, podendo inclusive existir liderança sem que exista um líder (Sutherland et al., 2014), rompendo com a necessidade atribuída ao líder individual para a materialização da liderança presente nas abordagens mainstream com seus heróis. Liderança "does not exist within a person, or even within a relationship between bounded figures called leaders and followers. Instead, leadership represents a kind of epiphenomenon that organizes and determines our experience of social reality and our experience of ourselves" (Kelly, 2014, p. 908). Desse modo, liderança é deslocada de um fenômeno sobre atores individuais para uma forma de organização social.

Assim, poder e autoridade não estão na mão de um indivíduo, mas são compartilhados, constituindo liderança como uma micropolítica (Kelly, 2014), tornando "leadership into a social reality rather than a physical one [...]" (Kelly, 2014, p. 911), rompendo com a concepção de liderança como um objeto com agência própria, provocando um movimento de descentramento que possibilita compreender liderança como uma forma de organizar um espaço interpretativo de ações e responsabilidades coletivas (Raelin, 2011).

Nas abordagens mainstream, os líderes, por meio de seu carisma, seus comportamentos e suas atitudes, moldam liderança, estabelecendo-a como um fenômeno individual, enquanto no pós-estruturalismo liderança é um fenômeno social e discursivo (Kelly, 2014). Liderança "is a process between rather than discrete entities either side that characterizes the ontological structure of leadership" (Kelly, 2014, p. 909). Assim, liderança é "a set of multiple and changing practices, pragmatically deployed by organizational subjects to re/draw alliances and, ultimately, exercise power" (Mackillop, 2018, p. 206). A dinâmica entre essas práticas permite que liderança não dependa da figura de um líder para existir, ou seja, "just because an organisation is leaderless, it does not necessarily mean that it is also leaderhipless" (Sutherland et al., 2014, p. 759). Por exemplo, Sutherland et al. (2014), ao analisarem movimentos sociais, demonstram como essas organizações apresentam liderança, 
apesar de não terem fisicamente um líder, demonstrando que não é necessário haver um líder para que exista liderança. Por estar fundamentado ontologicamente na crença de que somente existe liderança se houver líder, o mainstream não consegue explicar o processo de liderança dos diversos movimentos sociais que emergiram nos últimos anos, como a Primavera Árabe, o movimento Occupy ou as manifestações 20 centavos no Brasil. O pós-estruturalismo, ao descentrar o líder do processo de liderança, oferece uma ontologia que possibilita compreender esses fenômenos contemporâneos.

\section{CONSIDERAÇÕES FINAIS}

Liderança se torna um tema ainda mais relevante no contexto atual em que democracias estão em risco. Western (2008, p. 21) salienta que "critical theorists must go beyond identifying 'bad leadership practice' and aim to create and support successful ethical frameworks for leadership". Assim, este artigo buscou analisar como o pós-estruturalismo pode contribuir para a formação de um paradigma ético sobre liderança, analisando seus aspectos ontológicos. Ao considerar que poder e liderança não são propriedades dos líderes, rompendo com a lógica unidirecional top-down do mainstream que desconsidera agência, resistência e autonomia dos demais membros organizacionais, o pós-estruturalismo concebe liderança como um fenômeno relacional e multidirecional. A ontologia de liderança no pós-estruturalismo se alinha aos valores ocidentais de democracia, a fim de promover um mundo melhor (Kelly, 2014). Dessa forma, o pós-estruturalismo evidencia a complexidade das relações de poder, resistência e conflito (MacKillop, 2018), ressignificando o conceito de liderança como um espaço de "absent presence through which individual and collective desires for leadership are given expressions" (Kelly, 2014, p. 905), rompendo com a fronteira dualística líder versus seguidor (Collinson, 2011), afastando-se das análises de indivíduos heroicos.

A maioria dos trabalhos sobre liderança ainda estão relacionados às abordagens de traços e carismática/transformacional, buscando evidenciar contos de heróis individuais e habilidades carismáticas e transacionais dos líderes que promovem a efetiva mudança organizacional (Dinh et al., 2014; Herold, Fedor, Caldwell \& Liu, 2008). O pós-estruturalismo se contrapõe a essa visão de liderança ao evidenciar que liderança não está na mão de um indivíduo, concebendo liderança como um processo social multidirecional e mutuamente construído por múltiplos atores da organização, não tendo como ponto de partida os traços ou o carisma de um líder (Kelly, 2014). Para o pós-estruturalismo, liderança é uma micropolítica que organiza ações e responsabilidades coletivas (Raelin, 2011), e por isso deve ser compreendida no contexto em que é analisada. O contexto social e cultural formata as dinâmicas da liderança de acordo com suas especificidades. Portanto, liderança é uma construção discursiva histórica situada em determinado tempo e espaço, havendo necessidade de se considerar o contexto cultural e social, evidenciando as relações de poder pelas quais as identidades e a materialidade de liderança são construídas. Assim, o discurso adquire relevância no pós-estruturalismo para que se possa compreender como sentidos e significados sobre liderança são construídos e materializados.

O pós-estruturalismo - ao focar na análise de micropolíticas identitárias e discursivas para compreender liderança, e ao considerar liderança um fenômeno local e temporal - possibilita a emergência de diversas e diferentes definições sobre o que é liderança conforme o contexto analisado, caracterizando liderança como um significante vazio. Contudo, considerar liderança um significante vazio não enfraquece ou elimina o fenômeno em si, pois incompletude e fragmentação dão longevidade aos discursos de liderança. Dessa forma, liderança não é um objeto ou uma entidade, mas um processo de becoming que pode adquirir várias formas e envolver diversos atores (Kelly, 2014). Liderança é um fenômeno discursivo socialmente construído por meio de interações e negociações constantes em um processo de criação de sentidos e realidades que envolvem saberes e poderes (Collinson, 2011).

Por fim, em termos de limitações, este artigo fez uma análise crítica sobre liderança utilizando, para isso, o pós-estruturalismo, um dos possíveis estudos críticos de liderança (ECL), para se compreender criticamente esse fenômeno. Há outras abordagens críticas que possibilitariam uma releitura da ontologia mainstream sobre liderança, tais como marxismo, anarquismo, feminismo e teoria do processo de trabalho. Em relação a uma agenda futura de pesquisa pós-estruturalista sobre liderança, torna-se relevante o desenvolvimento de pesquisas empíricas em outras formas organizacionais, além das desenvolvidas em movimentos sociais, para se compreender em outros tipos de organizações o processo pelo qual liderança se estabelece como uma micropolítica que não depende da existência da figura de um líder. Ademais, sendo liderança um processo embodied, existe ainda uma lacuna de pesquisas sobre práticas corporais relacionadas à liderança em corpos transgêneros e de pessoas com deficiência. 


\section{REFERÊNCIAS}

Aarum A.J., \& Hansson, P. H. (2011). At the end of the road? On differences between women and men in leadership behaviour. Leadership \& Organization Development Journal, 32(5), 428-441.

Alvesson, M. (2002). Understanding organizational culture. London, UK: Sage.

Alvesson, M., \& Spicer, A. (2012). Critical leadership studies: the case for critical performativity. Human Relations, 65(3), 367-390.

Alvesson, M., \& Willmott, H. (2003). Introduction. In M. Alvesson, \& H. Willmott (Eds.), Studying Management Critically (pp. 1-22). London, UK: Sage.

Ayman, R., Korabik, K., \& Morris, S. (2009). Is transformational leadership always perceived as effective? Male subordinates' devaluation of female transformational leaders. Journal of Applied Social Psychology, 39(4), 852-879.

Boje, D. M., Alvarez, R. C., \& Schooling, B. (2001). Reclaiming story in organisation: narratologies and action sciences. In R. Westwood, \& S. Linstead (Eds.), The language of organisation (pp. 132-175). London, UK: Sage.

Calás, M., \& Smirchich, L. (1991). Voicing seduction to silence leadership. Organization Studies, 12(4), 567-602.

Carroll, B., Firth, J., Ford, J., \& Taylor, S. (2018). The social construction of leadership studies: representations of rigour and relevance in textbooks. Leadership, 14(2), 159-178.

Collinson, D. L. (2003). Identities and Insecurities: Selves at Work. Organization, 10(3), 527-547.

Collinson, D. L. (2006). Rethinking followership: a post-structuralism analysis of follower identities. Leadership Quarterly, 17(2), 179-189.

Collinson, D. L. (2011). Critical Leadership Studies. In D. Bryman, K. Collinson, B. Grint, B. Jackson, \& M. Uhl-Bien (Eds.), The Sage Handbook of Leadership (pp. 179-192). London, UK: Sage.

Collinson, D. L. (2014). Dichotomies, dialectics and dilemmas: new directions for critical leadership studies? Leadership, 10(1), 36-55.

Collinson, D. L., \& Collinson, M. (2009). Blended leadership: employee perspectives on effective leadership in the UK further education sector. Leadership, 5(3), 365-380.

Collinson, D. L., \& Tourish, D. (2015). Teaching leadership critically: new directions for leadership pedagogy. Academy of Management Learning \& Education, 14(4), 576-594.

Davies, J., Yarrow, E., \& Syed, J. (2020). The curious under-representation of women impact case leaders: can we disengender inequality regimes? Gender, Work \& Organization, 27(2), 129-148.

Delbridge, R., \& Ezzamel, M. (2005). The Strength of Difference: Contemporary Conceptions of Control. Organization, 12(5), 603-618.

Dinh, J. E., Lord, R. G., Gardner, W. L., Meuser, J. D., Liden, R. C., ... $\mathrm{Hu}, \mathrm{J}$. (2014). Leadership theory and research in the new millennium: current theoretical trends and changing perspectives. The Leadership Quarterly, 25(1), 36-62.
Eagly, A. H., \& Johannesen-Schmidt, M. C. (2001). The leadership styles of women and men. Journal of Social Issues, 57(4), 781-797.

Fairhust, G., \& Grant, D. (2010). The social construction of leadership: a sailing guide. Management Communication Quarterly, 24(2), 171-210.

Fletcher, J. K. (2004). The paradox of postheroic leadership: an essay on gender, power, and transformational change. The Leadership Quarterly, 15(5), 647-661.

Ford, J. (2005). Examining leadership through critical feminist readings. Journal of Health Organization and Management, 19(3), 236-251.

Ford, J. (2006). Discourses of leadership: gender, identity and contradiction in a UK public sector organization. Leadership, 2(1), 77-99.

Ford, J. (2010). Studying leadership critically: a psychosocial lens on leadership identities. Leadership, 6(1), 47-65.

Ford, J., \& Harding, N. (2007). Move over management: we are all leaders now. Management Learning, 38(5), 475-493.

Ford, J., Harding, N., \& Learmonth, M. (2008). Leadership as identity: constructions and deconstructions. Basingstoke, UK: Palgrave Macmillan.

Ford, J., Harding, N. H., Gilmore, S., \& Richardson, S. (2017). Becoming the leader: leadership as material presence. Organization Studies, 38(11), 1553-1571.

Foucault, M. (1974). The archeology of knowledge. London, UK: Tavistok.

Gerhardi, S. (1995). Gender, symbolism and organizational cultures. London, UK: Sage.

Gipson, A. N., Pfaff D. L., Mendelsohn, D. B., Catenacci, L. T., \& Burke, W. W. (2017). Women and leadership: selection, development, leadership style, and performance. The Journal of Applied Behavioral Science, 53(1), 32-65.

Grint, K. (2005). Leadership: limits and possibilities (Management, Work and Organisations). Basingstoke, UK: Palgrave Macmillan.

Herold, D. M., Fedor, D. B., Caldwell, S., \& Liu, Y. (2008). The effects of transformational and change leadership on employees' commitment to a change: a multilevel study. Journal of Applied Psychology, 93(2), 346-357.

Huczynski, A. (1993). Management gurus: what makes them and how to become one. London, UK: Routledge.

Kelly, S. (2014). Towards a negative ontology of leadership. Human Relations, 67(8), 905-922.

Kuipers, B. S., Higgs, M., Kickert, W., Tummers, L., Grandia, J., ... Van Der Voet, J. (2014). The management of change in public organizations: a literature review. Public Administration, 92(1), 1-20.

MacKillop, E. (2018). Leadership in organisational change: a poststructuralist research agenda. Organization, 25(2), 205-222.

Marshall, J. (2007). The gendering of leadership in corporate social responsibility. Journal of Organizational Change Management, 20(2), 165-181. 
Northouse, P. G. (2016). Leadership: theory and practice. Los Angeles, CA: Sage.

Ospina, S. M., Foldy, E. G., Fairhurst, G. T., \& Jackson, B. (2020). Collective dimensions of leadership: connecting theory and method. Human Relations, 73(4), 441-463.

Pullen, A., \& Vacchani, S. (2013). The materiality of leadership. Leadership, 9(3), 315-319.

Raelin, J. (2011). From leadership-as-practice to leaderful practice. Leadership, 7(2), 195-211.

Shamir, B., House, R. J., \& Arthur, M. (1993). The motivational effects of charismatic leadership: a self-concept based theory. Organization Science, 4(4), 577-594.

Spector, B. (2016). Carlyle, Freud and the great man theory more fully considered. Leadership, 12(2), 250-260.

Spicer, A., \& Alvesson, M. (2011). Conclusion. In M. Alvesson \& A. Spicer (Eds.), Metaphors we lead by: understanding leadership in the real world (pp. 194-205). Oxford, UK: Oxford University Press.
Sutherland, N., Land, C., \& Böhm, S. (2014). Anti-leaders(hip) in Social Movement Organizations: The case of autonomous grassroots groups. Organization, 21(6), 759-781.

Thomas, R., \& Davies, A. (2005a). What have the feminists done for us? Feminist theory and organizational resistance. Organization, 12(5), 711-740.

Thomas, R., \& Davies, A. (2005b). Theorizing the micro-politics of resistance: new public management and managerial identities in the UK public services. Organization Studies, 26(5), 683-706.

Toegel, G., \& Conger, J. A. (2002). A story of missed opportunities: qualitative methods for leadership research and practice. In K. Parry, \& J. Meindl (Eds.), Grounding leadership theory and research: issues, perspectives and methods (pp. 175-198). Greenwich, UK: Information Age Publishing.

Western, S. (2008). Leadership: a critical text. London, UK: Sage.

Wood, M. (2005). The fallacy of misplaced leadership. Journal of Management Studies, 42(6), 1101-1121.

Yukl, G. (2013). Leadership in organizations: global edition (8a ed). New York, NY: Pearson. 